\title{
Inserción extraordinaria del clítico lo de objeto directo en el español hablado por guaymíes costarricenses: notas preliminares
}

\section{Extraordinary Insertion of Direct Object Clitic lo in Spanish Spoken by Costa Rican Guaymies: Preliminary Remarks}

\author{
Rebeca Marín Esquivel \\ Universidad Nacional de Costa Rica \\ rebekme@gmail.com \\ https://orcid.org/0000-0002-3072-024X \\ (c) (1) (3) (2) \\ (c) UNAN-Managua \\ Recibido: marzo 2020. Aceptado: junio 2020 \\ DOI https://doi.org/10.5377/rll.v6i1.10126
}

\section{RESUMEN}

El objetivo de este artículo es explicar la inserción extraordinaria de clíticos del tipo 'lo voy a pensarlo', como posible resultado estructural del influjo de la lengua guaymí originaria de este grupo étnico, para lo cual, se recogió y se transcribió material oral de dieciocho hablantes bilingües de guaymí y español. Debido a que el análisis posee carácter exploratorio y revelador del fenómeno en cuestión, no se pretende llegar a conclusiones finales, sino únicamente proponer una explicación tentativa desde la perspectiva del contacto de lenguas.

Palabras clave: guaymí, sistema, pronombres, bilingüismo, contacto.

\begin{abstract}
SUMMARY
The objective of this article is to explain the extraordinary insertion of clitics of the type 'I will think about it', as a possible structural result of the influence of the Guaymí language originating in this ethnic group. To this purpose, oral material from eighteen bilingual speakers of Guaymí and Spanish was collected and transcribed. Due to the exploratory nature of the phenomenon under study, findings are not presented as conclusive, but rather as a tentative explanation from the perspective of language contact.
\end{abstract}

Key words: guaymi, system, pronouns, bilingualism, contact.

\section{INTRODUCCIÓN}

Los contactos lingüísticos que abundan en la área amerindia forman parte de la realidad lingüística diversa y característica de esta zona (Zimmerman, 1995; Zimmerman y Stolz, 2001). Así lo han comprobado Constenla (1991), Palacios (2008) y Quesada (2008a) para las áreas mesoamericana, peruana y amazónica, donde se albergan lenguas amerindias (de tan distintas familias lingüísticas), las cuales conviven con el español hablado por individuos de etnia indígena. Según Palacios (2002, 2005 y 2011), los rasgos del español hispanoamericano en situaciones de bilingüismo social surgen como resultados estructurales del influjo directo o indirecto de la lengua amerindia ( $p$. e. las discordancias 
de género y número en la frase nominal del tipo los personas, entre otros fenómenos). Estos rasgos, en muchas circunstancias, han llegado a tener contigüidad histórica entre las variedades del español de distintas generaciones de hablantes, de modo que su fosilización en el español de los padres bilingües con lengua indígena como L1 hace posible que sus descendientes crezcan expuestos a ese input del español y adquieran tales rasgos.

En reconocidos estudios como los de Flores (2008), García (2006) y Guillán (2012), se han documentado rasgos del español en contacto lenguas amerindias que incluyen, por ejemplo, cambios en la morfología nominal, transferencias fonéticas y reestructuración del sistema de clíticos ${ }^{1}$. Sin embargo, pese a los esfuerzos realizados para alcanzar un conocimiento exhaustivo sobre el español hablado por indígenas, los estudios siguen siendo escasos dada la falta de más descripciones sobre variedades del español circunscritas a situaciones de bilingüismo social en zonas indígenas.

Dentro de los pocos estudios que se han desarrollado en Costa Rica sobre contacto lingüístico, se aborda la posible influencia de las lenguas vernáculas en el español (Lininger, 1991; Constenla, 2002; Sánchez, 2012, 2013, 2015; Quesada, 2008 y 2009; Marín 2017), así como el desplazamiento de las lenguas en peligro (Portilla, 1986; Sánchez, 2012). Quesada (2008) explica que la influencia de las lenguas indígenas en las distintas variedades de español costarricense (hablado por indígenas) forma parte del contexto amerindio en el que ocurren diversas situaciones de contacto entre lenguas.

En lo relativo al sistema pronominal átono de tercera persona de objeto directo, específicamente en la variedad hablada por guaymíes costarricenses, Marín (2017) identifica una tendencia cuantitativa mucho más alta del pronombre lo que de la, las o los independientemente del género y número del referente (p.e. en lo iba a dejar a las hermanas), por lo cual se trataría de una neutralización de las categorías de género y número en favor del clítico lo. Esto resulta sintomático de la simplificación de este sistema, y por ello, del cambio (indirecto) inducido por contacto entre el español y el guaymí producto del mecanismo de convergencia lingüística².

Ahora bien, un fenómeno presente en este sistema pronominal del español guaymí corresponde a la producción de oraciones simples, cuyo uso del lo neutralizado aparece tanto en posición proclítica

1 Un ejemplo concreto sobre cambios en la morfología nominal documentado por Flores (2008) corresponde al uso del diminutivo chiquito en vez de chico probablemente por influencia semántica de la palabra náhualt tsitsiikitoon 'chiquito'. Las transferencias fonéticas y la reestructuración del sistema de clíticos se documenta para todas las variedades amerindias de español en contacto, por ejemplo, la pronunciación de la africada lateral sorda en el español guatemalteco, escrito ortográficamente como $t l$ (García, 2008), así como la reducción de la cantidad de clíticos en el sistema de tercera persona de objeto directo en el español del área andina en contacto con el guaraní y otras lenguas (Guillán, 2012).

2 En párrafos posteriores, se explica la definición de cambios directos e indirectos inducidos por contacto de lenguas de acuerdo con Palacios (2011) 
como enclítica, p. e. en lo voy a soltarlo (a los perros) y, aunque se desconoce las causas detonantes del empleo del doble clítico, lo cierto es que el guaymí como lengua de contacto podría estar influyendo en un resultado estructural de tal tipo ${ }^{3}$. Desde este panorama, en el presente artículo se analiza la inserción extraordinaria de clíticos del tipo lo voy a pensarlo, como posible resultado estructural del influjo (directo o indirecto) de la lengua guaymí originaria de este grupo étnico. Si bien las conclusiones a las que lleguemos tendrán un carácter muy preliminar, al menos sentarán los precedentes para la realización de futuros estudios en torno a este fenómeno.

En el marco de la Lingüística del Contacto se estudian las variaciones y cambios lingüísticos producto del contacto entre dos lenguas o más, así como de los resultados estructurales, sociolingüísticos, pragmáticos y léxicos derivados del influjo de una lengua en otra (Kaufman y Thomason, 1988; Apple y Muysken, 1996; Thomason, 2001; Matras, 2009). Con base en observaciones sobre los contactos entre el español y lenguas amerindias, Palacios (2002, 2010 y 2011) explica los fenómenos del español hablado por indígenas en distintos escenarios sociolingüísticos tomando en consideración que los miembros de comunidades bilingües, en su intento por lograr una comunicación efectiva con sus interlocutores, suelen utilizar procesos creativos en la producción de rasgos morfosintácticos novedosos, muchos de los cuales se presentan en individuos bilingües y monolingües (estos últimos expuestos al input de los primeros).

Debido a que estos fenómenos ocurren como resultado de procesos dinámicos de variación y cambio lingüístico, Palacios (2011), consecuente con los planteamientos de Thomason (2001, 2007 y 2010), propone que se aborden como cambios inducidos por contacto. Estos cambios pueden ocurrir únicamente en idiolectos particulares o extenderse, de manera sistemática y permanente, a toda una comunidad. Así las cosas, los rasgos de la modalidad de habla bilingüe suele estar relacionado con el tipo de bilingüismo, pues cuanto mayor sea el dominio que el hablante tenga de la L1 (p.e. lengua indígena), más resultados estructurales conformarán su variedad de L2 (p. e. el español). Obsérvese en el continuum de la Figura 1, la relación descrita entre modalidades de habla bilingües y tipos de bilingüismo:

En este continuum, se representa el carácter difuso del límite entre cada modalidad de bilingüismo consecutivo o simétrico (ubicadas en el centro de la figura), y el de las modalidades monolingües de español o lengua indígena (ubicadas en los extremos). La existencia de esos límites difusos hace posible identificar los rasgos gramaticales producto del contacto que pueden emerger en el habla de

3 A pesar Merma (2007) ha documentado el uso de doble clítico (p. e. lo iba a martarlo) para las variedades del español peruano en contacto con lenguas indoamericanas, las explicaciones del por qué, cómo y en qué modalidades de hablas ocurre este fenómeno pueden variar según las propiedades gramaticales de la lengua influyente y de su grado de conservación sociolingüística. 
bilingües consecutivos, y posteriormente, extenderse a bilingües simétricos, incluso a monolingües de español.

\begin{tabular}{|cccc|}
\hline Monolingües $\leftarrow$ bilingües consecutivo $\leftarrow$ & $\rightarrow$ bilingües simétrico & $\rightarrow$ \\
& $\downarrow$ & monolingües \\
$\downarrow$ & $\downarrow$ & $\downarrow$ \\
lengua indígena & $\mathrm{L}$ 1 lengua indígena & $\mathrm{L} 1=\mathrm{L} 2$ & español \\
& & & \\
\hline
\end{tabular}

Figura 1. Continuum de modalidades de habla bilingüe. Tomado de Palacios (2011, p. 20).

De esta forma, por medio de la clasificación de modalidades bilingües observadas en la figura 1, Palacios (2011) ha logrado explicar los efectos de la variación y el cambio en las variedades del español en contacto con lenguas indoamericanas bajo la denominación de cambios directos e indirectos. Se entiende por cambios directos a las importaciones de una lengua a otra de material léxico, funcional y estructural (Palacios, 2011); tal es el caso de la petición no entiendo este juego; por favor dame saliendo 'ayúdame a salir del juego' formada sobre la base de 'dar' + gerundio del español ecuatoriano usado por hablantes bilingües español-quechua.

Por su parte, los cambios indirectos se originan mediante la convergencia entre la evolución interna de la lengua influida ( $p$. e. el español) y las propiedades gramaticales de la lengua influyente (p. e. el quechua), de tal modo que estos cambios no pueden ser considerados como importaciones directas de material lingüístico, sino reinterpretaciones de una variación ya existente. Ejemplo de cambio indirecto se observa en la neutralización del sistema pronominal átono de objeto directo del español en favor de lo, fenómeno extendido entre individuos bilingües y monolingües de contextos indoamericanos, en el cual se aprovecha la disponibilidad de los clíticos a entrar en variación para potenciar que este sistema pronominal se simplifique.

\section{MATERIAL Y MÉTODOS}

Se realizó un muestreo en cadena a partir de un hablante de la comunidad quien contactó a otros miembros. Cuando se obtuvo un tope de 32 individuos, se aprovechó la posibilidad de hacer una 
selección por muestreo "intencionado" o "predeterminado" (Silva-Corvalán, 2001), de ahí que se escogieran 18 individuos guaymíes masculinos con bilingüismo consecutivo (guaymí como L1 y español como L2) para conformar grupos etiquetados como A, B y C. En el grupo A, se ubican los hablantes pertenecientes al rango de 53 a 63 años de edad; los del grupo $B$, poseen edades de entre 37 a 47 años, y los del grupo C, entran en el rango etario de 23 a 33 años.

Asimismo, se recogió material oral por medio de una entrevista semiestructurada que se aplicó a cada uno de los dieciocho individuos de la muestra ${ }^{4}$. En esta entrevista, los participantes compartieron tanto información sobre sus biografías lingüísticas (las lenguas que hablaban cuando eran niños, la lengua hablada por sus padres, etc.) como narraciones sobre temáticas libres. En segundo lugar, se organizó un corpus basado en cláusulas simples con uno o varios pronombres átonos de tercera persona de objeto directo según las propiedades gramaticales de género y número: lo, los, la y las, incluyendo las estructuras con clíticos dobles y sin estos. En tercer lugar, se cuantificaron las cláusulas haciendo exclusión de cláusulas cuyos referentes fueran ambiguos. Y, en cuarto lugar, se indagó en los rasgos morfosintácticos del guaymí que podrían motivar directa o indirectamente el uso de determinados clíticos en cláusulas particulares.

\section{RESULTADOS Y DISCUSIÓN}

A continuación, se describe el comportamiento cuantitativo de los clíticos de tercera persona de objeto directo lo y la en cuanto a la presencia o ausencia de clíticos dobles (p. ej. lo voy a pensarlo vs lo voy a pensar o voy a pensarlo), lo cual nos permitirán trazar las dimensiones de la variación lingüística que podría constituir el indicio de un cambio inducido por contacto. Todos los valores numéricos que aparecen en la tabla 1 se representan en números absolutos, cuya abreviatura es Abs. Al lado abajo de esta, se explica la interpretación de los datos tomando en cuenta que el total de cada categoría se expresa como divisor a la derecha de la barra simple (): p. e., la cantidad de 2/5 casos de clíticos dobles de lo, se debe leer como "dos clíticos dobles de un total de cinco casos exclusivamente para $10^{\prime \prime}$.

En el grupo A, el pronombre lo ocurre en 45/211 ocasiones con clítico doble y 166/211 sin este, mientras que la totalidad de apariciones de los (11/11), la (16/16) y las (3/3) se presentan sin clítico doble. Para el grupo B, lo se presenta 124/153 apariciones en cláusulas con un único clítico y 29/153 en cláusulas con doblado, en tanto los, la y las ocurren sin doble pronombre en 4/4, 19/19 y 2/2

4 Cada entrevista individual ronda entre cuarenta minutos y una hora aproximadamente. 
oportunidades respectivamente. En cuanto al grupo C, el clítico lo aparece en 10/123 ocasiones sin doblado y $113 / 123$ con este, en cambio los se presenta en $5 / 5$ oportunidades, la en 18/18 y las en 5/5

Tabla 1.

Apariciones de los clíticos dobles de objeto directo

\begin{tabular}{|c|c|c|c|c|}
\hline \multirow{2}{*}{ Grupos } & \multirow{2}{*}{ Clíticos } & Con clíticos dobles & Sin clíticos dobles & Total \\
\hline & & Abs. & Abs. & Abs. \\
\hline \multirow{4}{*}{$A$} & lo & 45 & 166 & 211 \\
\hline & los & 0 & 11 & 11 \\
\hline & la & 0 & 16 & 16 \\
\hline & las & 0 & 3 & 3 \\
\hline \multirow{4}{*}{ B } & lo & 29 & 124 & 153 \\
\hline & los & 0 & 4 & 4 \\
\hline & la & 0 & 19 & 19 \\
\hline & las & 0 & 2 & 2 \\
\hline \multirow{4}{*}{ C } & lo & 10 & 113 & 123 \\
\hline & los & 0 & 5 & 5 \\
\hline & la & 0 & 18 & 18 \\
\hline & las & 0 & 5 & 5 \\
\hline \multicolumn{2}{|c|}{ Total } & 84 & 486 & 570 \\
\hline
\end{tabular}

Como se observa, la aparición de clíticos dobles se registra únicamente para clíticos con género masculino en todos los grupos generacionales. Además, nótese la disminución cuantitativa en el empleo del doble lo registrado para el grupo A con 45 apariciones, 29 para el B, y 10 para el C, lo cual arroja luz sobre la pérdida paulatina de este fenómeno entre los hablantes entrevistados de cada grupo, quienes comparten el bilingüismo consecutivo guaymí-español con mayor dominio de guaymí (L1). Estos resultados nos motivan a explorar la producción de clíticos dobles en dos sentidos: uno de carácter lingüístico, en el que guaymí como lengua de contacto puede estar influyendo directa o indirectamente en la conformación de este fenómeno, y otro, de carácter sociolingüístico, en el que el factor de rango generacional se encuentra relacionado con su mayor empleo en hablantes mayores y su desempleo progresivo en individuos jóvenes.

En primer lugar, los clíticos dobles o doblados descritos hasta aquí, se explican a continuación como inserción extraordinaria de clítico. Este proceso consiste en agregarle a la estructura oracional un clítico 
extra que se consideraría innecesario en el marco del español costarricense sin contacto. Hay dos resultados finales. El efecto más visible de esa inserción extraordinaria de clítico son las oraciones con clíticos dobles, en las cuales coexisten un proclítico y un enclítico, ambos codificando la misma relación gramatical.

(1) Lo voy a soltarlo (a la mujer y al hombre).

(2) Lo vamo a castigarlo (al hombre).

El segundo efecto es la creación de estructuras de objeto reduplicado análogas a las oraciones de objeto reduplicado que se encuentran registradas para el español costarricense sin contacto y también encontradas en la variedad de español que nos ocupa. Este tipo de reduplicación de objeto ocurre solo en función de objeto directo, se utiliza la forma menos marcada lo, y no se presenta en las muestras orales de hablantes hispanocostarricenses (Quesada, 2009).

(3) Lo echó flor.

(4) Lo echó banano.

(5) Ahí donde lo conoció [que banano son alimenticia].

(6) ¿Cómo forma lo conocieron banano?

(7) Yo lo voy a agarrar día para usted.

(8) Yo lo agarré trabajo.

(9) Esa persona lo utiliza otro.

¿Cómo explicar esta inserción extraordinaria y sus efectos? Antes de ahondar en detalles, cabe aclarar que el guaymí es un lengua en cuyo sistema morfosintáctico, no existen los clíticos de ninguna persona y ni la concordancia de género gramatical, y, aunque posee marcación de plural -tre, esta se utiliza de manera muy intermitente y alaeatoria, por lo cual el hecho lingüístico analizado no corresponde a material importado del guaymí en el español, ya que es la lengua influida la que posee clíticos y no la lengua influyente.

En lo que a los objetos directos reduplicados compete, el clítico viene a reforzar una posibilidad estructural del español partiendo de un requerimiento estructural del guaymí, lengua en la cual el objeto directo y el verbo deben aparecer adyacentes (10) y en la que el objeto directo se coloca entre el 
auxiliar y el verbo principal en una perífrasis verbal con el orden AUX-verbo (11) (Quesada, 2008b; Murillo, 2016). Por consiguiente, como resultado de la convergencia entre ambas lenguas, el uso del proclítico estaría expresando también la función de guardar la unidad estructural OV del guaymí.

(10) Kwara gwe chibo kämi-ri tigre NOM cabra matar-P.REC

'El tigre mató a la cabra' (Murillo, 2016)

(11) kwe tä ümá d-en ñö te

3SG estar.PRS arena agarrar-PRS río en

'Él está agarrando arena en el río' (Murillo, 2016)

Para el caso de los clíticos dobles, la respuesta más probable a la pregunta anterior es que resulta de cumplir con el esquema sintáctico OV del guaymí (la inserción del proclítico), incluso en cláusulas que ya presentan un enclítico (p. ej. lo estaría pensándolo). Al parecer, la doble marcación podría tener, eventualmente, una función enfatizadora del objeto en el español guaymí. La marcación de relaciones pragmático-discursivas (énfasis, tópico, foco, etc.) mediante el uso de una partícula no es ajeno a las lenguas chibchas en general. Según Murillo (2016), el guaymí marca los sujetos con alta focalidad mediante la posposición gwe, y en boruca (Quesada, 2007) se hallan registrados los morfemas abí y ki, los cuales marcan topicalización., por lo que se podría estar transfiriendo este patrón a la variedad del español estudiada.

Por todo lo dicho, el fenómeno descrito constituye un cambio indirecto inducido por el contacto del guaymí con el español, puesto que se aprovecha la función del lo como codificador de caso acusativo, además de las posiciones proclítica y enclítica que puede tomar, con el propósito de crear una innovación gramatical que refuerce la posición del objeto directo antes de verbo como una estrategia para traspasar al español el requerimiento estructural del guaymí de preservar el esquema OV.

En segundo lugar, cabe preguntarse si resulta factible la preservación de este fenómeno a través de las generaciones de hablantes pese a la disminución cuantitativa del uso. Si bien nuestro estudio es muy exploratorio y no contamos con datos que nos permitan observar la proyección de tal cambio a largo plazo $^{5}$, resulta natural que el bilingüismo consecutivo de los hablantes de la muestra presente indicios

5 Según Thomason (2001), los cambios no cambios no se pueden predecir del todo, pues tomar una dirección u otra, dependiendo de los factores sociolingüísticos, como lo son el prestigio de la lengua para sus propios hablantes, los 
de gradualidad en relación con los estratos generacionales A, B y C. Desde esta perspectiva, es válido hipotetizar (por ahora) que los hablantes del grupo B podrían poseer menos dominio del guaymí (L1) que el de los individuos del grupo A, así como los del grupo B podrían poseer un dominio superior a los del grupo C. Esta correlación entre el mayor o menor dominio del guaymí y el rango generacional de los hablantes, podría ser la causa de que la aparición de oraciones con inserción extraordinaria de clítico lo disminuya en las generaciones más jóvenes.

\section{CONCLUSIONES}

La cuantificación del fenómeno denominado inserción extraordinaria de clítico lo arrojó luz sobre la existencia de este fenómeno en el español de los guaymíes entrevistados y su empleo en los tres grupos generacionales; sin embargo, nos permitió observar la disminución del uso en paralelo con rango etario, de modo que, mientras más jóvenes son los hablantes, menor es la tendencia a utilizar cláusulas del tipo lo voy a soltarlo.

Asimismo, la hipótesis propuesta en el apartado anterior, es que, desde un punto de vista lingüísticoestructural, el proclítico lo aparece en el español hablado por los guaymíes de la muestra, preservando así la relación indisoluble entre los elementos de orden OV. Esta relación viene a constituir un requerimiento estructural del guaymí que los bilingües consecutivos traspasan al español de manera indirecta, es decir, aprovechando los recursos morfosintácticos del español que son proclives a la variación y al cambio inducido por contacto.

Por supuesto, la viabilidad de estos resultados se debe comprobar en estudios futuros con una muestra poblacional representativa de la población total del guaymíes costarricenses y tomando en cuenta otros rasgos etarios que no hayan sido contemplados más arriba.

\section{REFERENCIAS}

Constenla, A. (2002). Algunos aspectos lingüísticos y socioculturales de la influencia de las lenguas indígenas en las variedades americanas del español, [en línea]. Disponible en http://lanicutexas.edu/.project/etext/lilas/cilla/constenla.pdf [Consulta: 2015, 13 de julio].

movimiento socioculturales, económicos, etc. Sien embargo, los estudios con muestra poblacionales muy representativas, suelen proyectar bastante bien el panorama de lo que podría ocurrir. 
Flores, J. (2008). México. En Palacios, A. (Coord.). El español en América. Contactos lingüísticos en Hispanoamérica (pp. 33-54). Barcelona: Ariel.

García, A. (2006). Contacto de lenguas en Guatemala: cambios en el sistema pronominal átono por contacto con la lengua maya. Revistas científicas de América Latina, El Caribe, España y Portugal, $15,11-71$.

García, A. (2008). Guatemala. En Palacios, A. (Coord.). El español en América. Contactos lingüísticos en Hispanoamérica (pp. 95-117). Barcelona: Ariel.

Guillán, M. (2012). Procesos de cambio lingüístico inducido por contacto en el español del Nea: El sistema pronominal átono. Tesis doctoral, Universidad Autónoma de Madrid, Madrid.

Marín, R. (2017). El sistema pronominal átono de tercera persona de objeto directo en el español hablado por guaymíes costarricenses. En Revista de Filología y Lingüística, 43 (2), 145-166.

Palacios, A. (2002). El sistema pronominal átono del español paraguayo: un caso de contacto de lenguas. En Calvo, J. (Coord). Teoría y práctica del contacto: el español en América en el candelero (pp. 123-143). Madrid: Vervuet- Iberoamericana.

Palacios, A. (2010). Algunas reflexiones en torno a la lingüística del contacto. ¿Existe el préstamo estructural? En Revista Internacional de Lingüística Iberoamericana (RILI), VIII (15), 33-55.

Palacios, A. (2011). Nuevas perspectivas en el estudio del cambio inducido por contacto: hacia un modelo dinámico del contacto de lenguas. Revista de Lenguas Modernas, 38, 17-36.

Portilla, M. (1986). Un caso de muerte de lenguas: el térraba. Estudios de Lingüística Chibcha, 5, 97246.

Quesada, M. A. (2008a). América Central. En Palacios, A. (Coord.). El español en América. Contactos lingüísticos en Hispanoamérica (pp. 57-69). Barcelona: Ariel.

Quesada, M. A. (2008b). Gramática de la lengua guaymí (ngäbe). Munchen: Lincom Europa.

Quesada, M. A. (2009). Historia de la lengua española en Costa Rica. San José: Editorial de Costa Rica.

Sánchez, C. (2012). El papel de la escuela en el desplazamiento y en la conservación de la lengua malecu. Revista Educación, 36 (1), 25-43.

Sánchez, C. (2013). Lenguas en peligro de Costa Rica: vitalidad, documentación y descripción. Káñina, 
XXXVII (1), 219-250.

Sánchez, C. (2015). El sistema pronominal átono de tercera persona en el español hablado por los malecus de Costa Rica. CLAP, 61, 79-103.Thomason, S. (2001). Language Contact. Edinburgh: University Press.

Stolz, T. y Zimmerman, T. (2001). Lo propio y lo ajeno en las lenguas austronésicas y amerindias. Procesos interculturales en el contacto de las lenguas indígenas con el español en el Pacífico e Hispanoamérica. Madrid: Iberoamericana.

Thomason, S. (2001). Language Contact. Edinburgh: University Press.

Thomason, S. (2007). Social and linguistic factors as predictors of contact-induced change.

Journal of language contact, 2, 2-56.

Thomason, S. (2010). Contact explanation in linguistics. En Hickey, R (eds.). The Handbook of language contact (pp. 31-47). Oxford: Whiley Blackwell.

Zimmermann, K. (1995). Aspectos teóricos y metodológicos del contacto de lenguas. En Zimmermann, K. (ed.): Lenguas en contacto en Hispanoamérica: Nuevos enfoques (pp.9- 34). Madrid/Frankfurt: Iberoamericana/Nervuert.

\section{Abreviaturas}

AUX auxiliar

NOM nominativo

OV objeto y verbo

p.e. por ejemplo

P.REC pasado reciente

PRS presente

SG singular 